\title{
ENZIMAS ANTIOXIDANTES EN CEREBRO DE COBAYOS NATIVOS DE LAS GRANDES ALTURAS
}

\author{
MÓNICA PAJUELO T'. , LAURA YAMAdA A.', EDGAR FLORENTINI R. ${ }^{2}$ Y ELIZABETH CARRANZA A. ${ }^{2}$ \\ 'DEPARTAMENTO ACADÉMICO DE BIOQUIMICA, FACULIAU UE FAKMACIA Y BIOQUIMICA, E 'INSTITUTO NACIONAL DE \\ BIOLOGIA ANDINA, FACULTAD DE MEDICINA, UNIVERSIDAD NACIONAL MAYOR DE SAN MARCOS
}

\begin{abstract}
RESUMEN
Se ha determinado la actividad de las enzimas antioxidantes Superóxido Dismutasa (SOD), Glutation Peroxidasa (GPx) y Catalasa (CAT), la concentración de malondialdehído (MDA) e hidroperóxidos como indicadores de peroxidación lipídica, y la actividad de fosfolipasa A, como mediador de la lipoperoxidación, en tejido cerebral de cobayos nacidos y criados en Cerro de Pasco a $4300 \mathrm{~m}$, en comparación con cobayos del nivel del mar (NM), con la finalidad de cstimar las diferencias entre ambos grupos. En la actividad de las enzimas antioxidantes no se hallaron diferencias significativas entre ambos grupos. En el grupo de estudio (ALT) se halló la actividad de fosfolipasa A y la concentración de hidroperóxidos significativamente mayores que en el grupo control ( $\mathrm{p}<0.01$ y $\mathrm{p}<0.001$, respectivamente). Asimismo, se encontró que la concentración de MDA es significativamente menor en el grupo de ALT ( $\mathrm{p}<0.01$ ). Por tanto, los datos observados presentan evidencia de que la altura no influiría en la actividad de las enzimas antioxidantes en tejido cerebral de cobayos, pero probablemente sí en el grado de lipoperoxidación, por una mayor actividad de fosfolipasa A y concentración de hidroperóxidos; sin embargo una menor concentración de MDA indicaría la participación de otros mecanismos antioxidantes.
\end{abstract}

Palabras clave: Lipoperoxidación, superóxido dismutasa (SOD), glutation peroxidasa (GPx), catalasa (CAT), malondialdehido (MDA), hidroperóxidos, fosfolipasa $A$.

\section{SUMMARY}

We have determined antioxidant enzyme activities of: Superoxide Dismutase (SOD), Glutathione Peroxidase (GPx) and Catalase (CAT); levels of malondialdehyde (MDA) and hydroperoxids as lipoperoxidation indicators, and phospholilpase activity as mediator of the lipoperoxidation in cerebral tissue of guinea pigs born and bred in Cerro de Pasco at $4300 \mathrm{~m}$ (ALT), with respect to guinea pigs at sea level (NM), in order to detect real differences between both groups. With respect to activity of antioxidant enzymes, we have not found significant differences between both groups. In the study group (ALT) we have found significant differences in the activity of phospholipase and level of hydroperoxides $(\mathrm{p}<0.01 \mathrm{y} p<0.001$. respectively) as compared to sea level group. Also, we have found that level of MDA is significantly less in the study group $(\mathrm{p}<0.01)$.Thus, observed data would lead us to say that altitude does not influence activity of antioxidant enzymes in cerebral tissue of guinea pigs, but it probably influences level of lipoperoxidation, due to a major activity of phospholipase and level of hydroperoxides; nevertheless, a minor level of MDA would indicate the participation of other antioxidant mechanisms.

Key words: Lipoperoxidation, superoxide dismutase (SOD), glutathione preoxidase (GPx), catalase (CAT), malondialdehyde (MDA), hydroperoxids, phospholipase.

\section{INTRODUCCIÓN}

El oxígeno, elemento esencial para la vida adquiere un carácter tóxico en nuestro organismo por la formación natural de especies químicas altamente reactivas como los radicales libres (RL), los cuales causan reacciones de óxido reducción en cadena, dañando diversas estructuras lipídicas, proteicas e incluso al ADN y ARN $(1,2,3,4)$. Estos RL son removidos de nuestro organismo por diversos mecanismos antioxidantes, principalmente por la acción de las enzimas antioxidantes Superóxido Dismutasa (SOD), Glutation Peroxidasa (GPO) y Catalasa (CAT), manteniendo un «equilibrio oxidativo" $(5,6)$. En ocasiones, las defensas antioxidantes no son suficientes para contrarrestar la acción de los RL y dan lugar al "estrés oxidativo". Este ha sido involucrado en diversos estados patológicos de tipo degenerativo, e incluso en los procesos naturales de envejecimiento y muerte $(7,8)$.

En el Perú, un considerable porcentaje de la población vive sobre los $3000 \mathrm{~m}$, ambiente en el que existe menor presión barométrica y por tanto, menor presión parcial de oxígeno. Esta condición, principalmente, ha llevado a que el organismo de diferentes seres vivos, entre ellos el hombre, se 
adapten y desarrollen mecanismos anatómicos, fisiológicos y bioquímicos, como lo demuestran estudios hasta ahora realizados $(9,10,11,12,13)$. Pero aún existe poca información sobre la influencia que ejerce la altura en el equilibrio oxidativo en el cerebro, órgano principal de los mamíferos, que contiene alto nivel de lípidus y calcio; asimismo, requiere altos niveles energéticos y un elevado consumo de oxígeno, por tanto es muy susceptible al daño oxidativo por lipoperoxidación (3).

El objetivo del presente trabajo es medir la actividad de las enzimas antioxidantes SOD, GPO y CAT y los productos intermedios y finales de la lipoperoxidación como son los hidroperóxidos y el malondialdehído (MDA) (14); también, la fosfolipasa A como mediador de la lipoperoxidación en cerebro de cobayos oriundos de Cerro de Pasco ( $4300 \mathrm{~m}$ ) y de Lima $(150 \mathrm{~m})$. con el fin de aportar información que permita un mejor conocimiento sobre la influencia que la altura ejerce sobre el equilibrio oxidativo en el cerebro, y contribuir así a la comprensión de los fenómenos bioquímicos en los habitantes de las grandes alturas.

\section{MATERIAL Y MÉTODOS}

Animales. Se emplearon cobayos (Cavia aparea) machos adultos criados a nivel del mar (Lima, 150 m) y en altura (Pasco, $4300 \mathrm{~m}$ ). Para el experimento se criaron 10 animales en altura y 10 a nivel del mar. Fucron alimentados ad libitum. Los animales criados en altura fueron llevados al Laboratorio del Instituto Nacional de Biología Andina en Morococha (4 $540 \mathrm{~m}) 30$ días antes de ser sacrificados. Los animales criados a nivel del mar fueron sacrificados en el I aboratorio del Instituto Nacional de Biología Andina en Lima. Las pruebas analíticas fucron llevadas a cabo en Lima.

Extracción y tratamiento de la muestra. Los animales fueron sacrificados por decapitación, e inmediatamente se procedió a retirar el cerebro por la parte frontal del cráneo. El cerebro fue lavado con solución de cloruro de sodio $0.9 \%$ helado, pesado e inmediatamente colocado en bolsa de polielileno identificada, y congelado en una mezcla de hielo seco con metanol $\left(-7^{\circ} \mathrm{C}\right)$. Las muestras de altura fueron llevadas a Lima, donde se procesaron junto con las muestras de nivel del mar. Cada cerebro fue dividido en tres partes, de aproximadamente 1 gramo cada una. Cada fracción fue homogenizada con $4 \mathrm{~mL}$ de su respectivo buffer en un baño de hielo y en un liempo no mayor de 5 minutos. Se utilizó buffer fosfato $50 \mathrm{mM} \mathrm{pH} 7.4$ y 2mercaptoetanol $0.001 \mathrm{M}$ para el análisis de SOD, GPx, CAT y proteínas; buffer Tris $20 \mathrm{mM}$ pH 7.6 para la determinación MDA, fosfolipasa A y proteínas y buffer fosfato salino $5 \mathrm{mM}(\mathrm{NaCl} 150$ $\mathrm{mM}) / \mathrm{BHT} 4 \mathrm{mM}$ pH 7.4 para el análisis de hidroperóxidos y grasa total.

a) Determinación de SOD. Se utilizó el kit comercial RANDOX, basado en el Método de Mc Cord and Fridovich (15). Este método emplea xantina y xantina oxidasa para formar radicales superóxido, éstos reaccionan con cloruro de 2(4-yodofenil)-3-(4-nitrofenol)-5-fenil tetrazolio (I.N.T.) para formar un colorante formazán rojo. La actividad de la enzima se mide por el grado de inhibición en la formación del colorante en la reacción. Una unidad de SOD fue definida como la cantidad de enzima necesaria que puede causar el $50 \%$ de inhibición del colorante a temperatura ambiente. La actividad enzimática se calculó usando un standard comercial, y los resultados se expresan como U/ mg proteína.

b) Determinación de GPx. Se utilizó el kit comercial RANDOX, eL método esta basado en el trabajo de Plagia y Valentine (16). La glutation peroxidasa cataliza la oxidación del glutation (GSH) por el hidropcróxido de cumeno. El glutation oxidado (GSSG) en presencia de la glutation reductasa y NADPH es inmediatamente convertido en su forma reducida (GSH), con una oxidación concomitante de NADPH en $\mathrm{NADP}^{+}$. Se mide la disminución de la absorbancia a $340 \mathrm{~nm}\left(\mathbf{e}=6.22 \mathrm{mM}^{-1}, \mathrm{~cm}^{-1}\right)$. Los resultados se expresan como U/ mg proteína.

c) Determinación de CAT. La actividad de la CAT fue estimada por el método de Aebi.(17). La descomposición enzimática del peróxido de lidirógeno $\left(\mathrm{H}_{2} \mathrm{O}_{2}\right)$ puede ser medida directamente por decrecimiento de la absorbancia a $240 \mathrm{~nm}$ $\left(e_{24 !}=0.00394 \pm 0.0002\right.$ litros $\left.\mathrm{mmol}^{-1} \mathrm{~mm}^{-1}\right)$. La actividad de la enzima fue determinada midiendo el consumo de $\mathrm{H}_{2} \mathrm{O}_{2}$ en un medio conteniendo $2.9 \mathrm{~mL}$ de sustrato $\left(\mathrm{H}_{2} \mathrm{O}, 0.05 \%\right.$ en buffer de fusfatos $50 \mathrm{mM} \mathrm{pH}$ 7. La absorbancia inicial de esta mezcla debe encontrarse en el rango de $(0.50-0.55)$ y $0.1 \mathrm{~mL}$ de sobrenadante diluido al $50 \%$ con buffer fosfato $\mathrm{pH} 7$. Una unidad es dufinida como la cantidad de enzima que puede descomponer un $\mu \mathrm{mol}$ de $\mathrm{H}_{2} \mathrm{O}_{2}$ por mirute a $25^{\circ} \mathrm{C}$ y a pH 7.0. Los resultados se expresan como $\mathrm{U} / \mathrm{mg}$ proteína.

d) Determinación de MDA. El contenido de MDA fue cvaluado como sustancias reactivas al ácido tiobarbitúrico (TBARS) mediante una técnica espectrofotométrica descrita en el trabajo de Fiong, Yeh, Chang y $\mathrm{Hu}$ (18), donde se adiciona $\mathrm{NaOH}$ para separar el MDA unido a las proteínas. Las sustancias reactantes con el ácido tiobarbitúrico fueron calculadas usando el 
coeficiente de extinción molar $1.56 \times 105 \mathrm{M}-1 \mathrm{x}$ $\mathrm{cm}-1$. Los resultados se expresan como mmoles/ mg proteína).

e) Determinación de Hidroperóxidos. El método utilizado fue el descrito por J. Nourooz - Zadeh, J. Tajaddini - Sarmadi y S. P. Wolf (19). Está basado en la oxidación de iones ferrosos a férricos por hidroperóxidos en condiciones ácidas. Los iones férricos se unen al indicador anaranjado de xilenol para formar un complejo estable coloreado, que puede ser leído a $560 \mathrm{~nm}$. El coeficiente de extinción molar aparente para una gran variedad de hidroperóxidos a $560 \mathrm{~nm}$ es $4.3 \times 104 \mathrm{M}^{-1} \times \mathrm{cm}^{-1}$. indicando que aproximadamente se produce 3 moles de ion férrico por mol de hidroperóxido adicionado. Para diferenciar los hidroperóxidos de otros peróxidos, se agregó trifenilfosfina (TPP), un reductor específico de los hidroperóxidos. Para el ensayo se preparó un extracto lipídico, para lo cual se midió $0.2 \mathrm{~mL}$ del homogenizado respectivo, al cual se adicionó $0.4 \mathrm{~mL}$ de agua y $0.5 \mathrm{~mL}$ de metanol; la mezcla fue agitada en vortex por aproximadamente 30 segundos. Se adicionó $1.0 \mathrm{~mL}$ de acetato de etilo y la suspensión fue agitada en vortex por 30 segundos más. La suspensión fue centrifugada a $3000 \mathrm{~g}$ por 10 minutos. La capa orgánica fue transferida a un tubo. Se adicionó $1.0 \mathrm{~mL}$ de acetato de etilo a la fase acuosa, fue mezclada en vortex por 30 segundos, centrifugada a $3000 \mathrm{~g}$ por 10 minutos. La capa orgánica fue transferida al tubo anterior y mezclados ambos extractos. En sendos tubos se midieron $0.200 \mathrm{~mL}$ del extracto lipídico, a uno de los cuales se le añadió 0.020 $\mathrm{mL}$ de TPP $10 \mathrm{mM}$ y al otro $0.020 \mathrm{~mL}$ de metanol; se dejaron en reposo por 30 minutos y luego se les añadió $1.8 \mathrm{~mL}$ del reactivo FOX (Sulfato de amonio ferroso $25 \mu \mathrm{M}$, anaranjado de xilenol $100 \mu \mathrm{M}$, Ácido Sulfúrico $25 \mathrm{mM}$, butil hidroxi tolueno (BHT) $4 \mathrm{mM}$ en $90 \% \mathrm{v} / \mathrm{v}$ de metanol HPLC). Se dejó en reposo 30 minutos y se leyó a $530 \mathrm{~nm}$ frente a un blanco que tiene TPP, metanol y reactivo FOX. La diferencia en la absorbancia de la muestra sin TPP y con TPP está en relación directa con la concentración de hidroperóxidos en la muestra. Los resultados se expresan como mmoles/g grasa total.

f) Determinación de Fosfolipasa A. Se utilizó el método de De Oliveira \& Palma. Este es un método espectrofotométrico basado en el cambio de $\mathrm{pH}$, debido a la liberación de ácidos grasos por acción de la fosfolipasa A sobre la fosfatidil colina. Este cambio de $\mathrm{pH}$ se evidencia por el cambio de absorbancia a $558 \mathrm{~nm}$ del rojo de fenol (20). En una cubeta de un $\mathrm{cm}$ de espesor colocar $0.4 \mathrm{~mL}$ de Tris $\mathrm{HCl} \mathrm{pH} 7.9(7.5 \mathrm{mM}), 0.08 \mathrm{~mL}$ de
Fosfatidilcolina disuelto en Triton X-100, $0.4 \mathrm{~mL}$ de Cloruro de Calcio $5 \mathrm{mM}$ y $0.04 \mathrm{~mL}$ de Rojo de fenol $0.8 \mathrm{mM}$. La reacción fue iniciada por la adición de $0.1 \mathrm{~mL}$ de sobrenadante (El homogenizado respectivo fue centrifugado a $1000 \mathrm{rpm} \times 10$ minutos). El decrecimiento de la absorbancia del rojo de fenol fue medido después de 5 minutos de incubación a $37^{\circ} \mathrm{C}$. La actividad se expresa como cambio de absorbancia por minuto por $\mathrm{mg}$ de proteína.

g) Otras determinaciones. Para la determinación de proteínas se utilizó el método Lowry (21) el cual se basa en la reducción del $\mathrm{Cu}^{2+} \mathrm{a} \mathrm{Cu}^{+}$por acción de las proteínas en medio alcalino. $\mathrm{El} \mathrm{Cu}^{+}$ y los aminoácidos aromáticos reducen al reactivo de Folin-Ciocalteau a heteropoli-molibdeno azul, el cual es medido a $500 \mathrm{~nm}$, y para la grasa total, el método de Bragdon (22) que se basa en la oxidación de los lípidos por efecto de la mezcla de bicromato de potasio - ácido sulfúrico y la medición del cromo reducido a $580 \mathrm{~nm}$.

Análisis Estadístico.Los resultados obtenidos de los grupos de estudio y de control fueron expresados en términos de promedios y desviaciones estándar. Para evaluar las diferencias entre ambos grupos se aplicó la prueba $t$ de student. Todo resultado cuyo valor de $\mathrm{p}$ es menor que 0.05 se considera significativo.

\section{RESULTADOS}

En la Tabla 1 se presentan los valores medios de la actividad de las enzimas antioxidantes expresados en $\mathrm{U} / \mathrm{mg}$ de proteína. Como puede apreciarse, los valores de la SOD, GPx y Cat en cerebros de cobayos nacidos en las grandes alturas no presentan diferencias significativas cuando se les compara con sus similares del nivel del mar.

En la tabla 2 se presentan los valores medios de la concentración de MDA expresadas en mmoles/ mg proteína. Al comparar ambos grupos observamos que el nivel de MDA en cerebro de cobayos oriundos de la altura son significativamente menores en relación con los cobayos del nivel del mar $(p<0.01)$.

En relación a la concentración de hidroperóxidos cerebrales, expresados en mmol/g grasa, se observa que existe diferencia significativa $(p<0.001)$ cuando se comparan ambos grupos de animales (Ver Tabla 2).

Al efectuar la comparación de las medias de la enzima fosfolipasa A expresada por D Abs/min/ mg proteína en cerebro de cobayos de altura y cerebro de cobayos de nivel del mar se aprecia que existe diferencia significativa ( $p<0.01$ ) cntrc ambos grupos (Ver Tabla 2). 
Tabla $N^{\circ} 1$ Niveles medios de Superóxido Dismutasa, Glutation Peroxidasa y Catalasa en cerebro de cobayos.

\begin{tabular}{|l|l|l|c|c|c|}
\hline & ALTTUD & N & Media & DS & p \\
\cline { 2 - 6 } Superóxido Dismutasa & Nivel del mar & 10 & 52.0 & 10.8 & n.s \\
(U/mg Proteína) & Altura. & 10 & 48.5 & 13.2 & \\
\hline Glutation Peroxidasa & Nivel del mar & 10 & 23.0 & 7.6 & \\
(umol/mg Proteína) & Altura & 10 & 27.8 & 5.2 & $\mathrm{n} . \mathrm{s}$ \\
\hline Catalasa & Nivel del mar & 10 & 853.3 & 111.4 & \\
(U/mg Proteína) & Altura & 10 & 903.7 & 142.8 & n.s \\
\hline
\end{tabular}

Tabla $\mathrm{N}^{\circ} 2$ Niveles medios de Malondialdehido, Hidroperóxidos y Fosfolipasa A en cerebro de cobayos

\begin{tabular}{|c|c|c|c|c|c|}
\hline & ALTITUD & $\mathrm{N}$ & Media & DS & $\mathrm{p}$ \\
\hline $\begin{array}{l}\text { Malondialdehido } \\
\text { (pmol/g Proteína) }\end{array}$ & $\begin{array}{l}\text { Nivel del mar } \\
\text { Altura }\end{array}$ & $\begin{array}{l}10 \\
10\end{array}$ & $\begin{array}{l}142.2 \\
103.1\end{array}$ & $\begin{array}{l}23.6 \\
25.2\end{array}$ & $<0.01$ \\
\hline $\begin{array}{l}\text { Hidroperóxidos } \\
\text { (mmol/g Grasa) }\end{array}$ & $\begin{array}{l}\text { Nivel del mar } \\
\text { Altura }\end{array}$ & $\begin{array}{l}10 \\
10\end{array}$ & $\begin{array}{l}1.0 \\
2.4\end{array}$ & $\begin{array}{l}0.3 \\
0.8\end{array}$ & $<0.001$ \\
\hline $\begin{array}{l}\text { Fosfolipasa A } \\
\text { (D Abs } / \mathrm{min} / \mathrm{mg} \text { Pro- } \\
\text { teina) } \times 10^{-3}\end{array}$ & $\begin{array}{l}\text { Nivel del mar } \\
\text { Altura }\end{array}$ & $\begin{array}{l}10 \\
10\end{array}$ & $\begin{array}{l}3.41 \\
6.16\end{array}$ & $\begin{array}{l}1.67 \\
2.53\end{array}$ & $<0.01$ \\
\hline
\end{tabular}

\section{Discusión}

La respuesta adaptativa al estrés oxidativo está referida a la capacidad del organismo a resistir los efectos de un agente oxidante, mediante la primera línea de defensa, conformada por las enzimas antioxidantes preventivas catalasa y glutation peroxidasa, que reducen la velocidad de iniciación de la cadena oxidativa, al reaccionar con hidroperóxidos, y el antioxidante interruptor de la cadena oxidativa, superóxido dismutasa, que actúa atrapando los radicales superóxido libres $(2,4)$. En nuestro estudio se ha encontrado que los niveles dc estas cnzimas en tejido cerebral de cobayos criados en altura son similares a los de cobayos criados a nivel del mar (no existen diferencias significativas), con esto se evidencia que en las grandes alturas, en un annbiente hipóxico, el tejido cerebral, no necesariamente responde con una mayor producción de enzimas antioxidantes, otros tejidos parecen comportarse en forma similar. Así en un estudio realizado por Agostoni y col (23). sobre niveles de enzimas antioxidantes en el eritrocito y de selenio sérico en una población andina $(3800 \mathrm{~m})$, menciona que no encontró diferencias significativas en las actividades de SOD y CAT, pero sí una reducción significativa en la actividad de GPX que lo asocia con una baja ingesta de selenio en la dieta de pobladores de altura.

La similitud de actividades de las enzimas encontradas en nuestro estudio, en ambos grupos, haría suponer que en un equilibrio oxidativo, no existirian diferencias significativas en los niveles de oxidación; sin embargo los niveles de hidroperóxidos y malondialdehído encontrados fueron significativamente diferentes, sugiriendo que los mecanismos de adaptación a la altura son diferentes.

Uno de estos mecanismos sería la despolarización de la gradiente de iones en la célula, incrementando la concentración de $\mathrm{Ca}^{++}$, lo cual va a activar una 
hidrólisis de fosfolípidos en la membrana celular $(24,25)$. Esta hidrólisis es mediada por la enzima fosfolipasa A, liberando una mayor cantidad de ácidos grasos libres. A partir de los ácidos grasos poliinsaturados, que contienen enlaces dobles de grupos metilenos interrumpidos, se generaria una mayor lipoperoxidación. En nuestro estudio se halló una mayor cantidad de fosfolipasa $A$, que siguiendo la cadena metabólica, incrementaría la producción de ácidos grasos libres, que a su vez, produciría un incremento de los niveles de hidroperóxidos.(24,25).

Otro mecanismo de adaptación a la altura involucrado sería que, al existir una menor presión parcial de oxígeno en el aire, genera un aumento en la concentración de hemoglobina en sangre y con ello, la cantidad de hierro, que es uno de los elementos que favorece la lipoperoxidación a través de la reacción de Fenton y Haber Weiss (26) incrementando los niveles de hidroperóxidos, tal como se observa en nuestros resultados.

La acumulación de grupos hidroperóxido en carbonos alílicos lleva a su vez a la ruptura de la cadena carbonada y a la formación de MDA (27). En el estudio realizado se aprecia que la cantidad de MDA en altura es menor que a nivel del mar, lo cual no guarda relación con los niveles de hidroperóxidos encontrados, posiblemente debido a la acción de otros mecanismos antioxidantes, como la Vitamina E (28), que evitaría la formación de una mayor cantidad de MDA gracias a la afinidad que posee con los fosfolípidos de la membrana mitocondrial, membrana del retículo endoplasmático y membrana plasmática. La Vitamina $E$ interrumpe las reacciones en cadena de los radicales mediante la transferencia de un $\mathrm{H}^{+}$fenólico a un radical peroxilo. También se puede señalar a la ceruloplasmina, proteína plasmática que evitaría la lipoperoxidación por la oxidación de $\mathrm{Fe}^{+2}$ a $\mathrm{Fe}^{+3}$, que en un estudio realizado por Vicente y Miñano, en sujetos de altura se encontró en mayor cantidad que en sujetos de nivel del mar ( $\mathrm{p}<0,0001)$.

Por lo expuesto, se podría indicar que la mayor cantidad de hidroperóxidos encontrados en tejido cerebral de cobayos de altura, frente a una menor formación de MDA, estaría mediada por mecanismos diferentes a la acción de las enzimas antioxidantes (SOD, CAT y GPx), por lo que la comparación de los niveles de otros antioxidantes no enzimáticos en tejido cerebral de cobayos contribuiría a esclarecer en cierta forma las diferencias significativas de productos de lipoperoxidación halladas en el presente estudio.

\section{REFERENCIAS BIBLIOGRÁFICAS}

1. Pryor, W. 1983. Oxyradicales and Related Species: Their Formations, Lifetime and reactions. Ann. Rev. Phys. 48: 657-667.

2. Mc Cord, J. 1983. The Superoxide free radical: Its biochemistry and pathophysiology. Surgery 94 (3): 412-414.

3. Tiskow G. 1996. Radicales Libres en Biología y Medicina: Una Breve Revision. Gac Cien Vet 1: 44-57.

4. Fridovich, I. 1995. Superoxido radical and Superoxide Dismutasa Ann. Rev. Biochem. 64: 97112.

5. Bruce S, McEwen. 1998. Protective and Damaging Effects of Stress Mediators. The New England Journal of Medicine 338 ( 3), 171-178.

6. Montero, M. 1996. Los Radicales Libres y las Defensas Antioxidantes. Revisión. Anales de la Facultad de Medicina. UNMSM. 57 (4), 278 281.

7. Marx J. L. 1987 Oxygen Free Radicals Linked to Many Diseases. Science 235: 529-531.

8. Kasapoglu M., Ozben T. 2001. Alterations of Antioxidants Enzymes and Oxidative Stress Markers in Aging. Exp Gerontol 36 (2), 209-220.

9. Boero J., Arregui, A., Rovainen, C. M., Woolsey, T. A.1996 Remodelling of Brain Capillaries during chronic hypoxia in mice. En: The Brain at High Altitude: Clinical Research an Molecular Physiology. Acta Andina 2 (Special Issue $\mathrm{Y}$ ).

10.Arregui, A. 1993. Migraine and Chronic Mountain Sickness: Epidemiological Studies. En León Velarde, F. y Arregui, A. Ed. Investigaciones Básicas y Clínicas. Homenajhe a C. Monge C. IFEA - UPCH.

11. Ward M. P. J. and West J.B. 1995. High Altitude Medicine and Physiology, 2da Ed. By Chapman \& Hall, London. UK, 1995.

12.Villena J. 1998 Cambios Metabólicos en la Hipoxia Crónica. Acta Andina 8: 95-103.

13. Bouverot P. 1985 Adaptation to AltitudeHypoxia in Vertebrates. Cap.6: «Biochemical Changes». Springer Vertag. Berlin Heidelberg. New York. Tokyo.

14.Valenzuela A. 1991. The Biological Significance of Malondialdehyde Determination in the Assessment of Tissue Oxidative Stress. Life Sciences 48: 301-309. 
15. Mc Cord, J. M and Fridovich, I. 1969. Superoxide Dismutase - an enzymatic function for erythrocuprein. J.B. C. 244 (22), 6049- 6053.

16. Paglia, D. E. and Valentine, W. N. 1967. Studies on thequantitative and qualitative characterization of erythrocyteglutathione peroxidase. J. Lab. Clin. Med.70, 158-168.

17. Aebi H. 1974. Catalase. In: Methods of Enzymatic Analysis(Bergmeyer HU, Ed), Verlag Chemie; Weinheim, pp 673-678.

18. Yu-Lun Hong, Shu-Lan Yeh, Chia-Yu Chang, MiaoLin $\mathrm{Hu}$. 2000. Total Plasma Malon-dialdehyde levels in 16 Taiwanese College Students determined by various Thiobarbituric acid test and an improved high-performance liquid chromatography-based method. Clinical Biochemistry $33(8) 619-625$.

19. Nourooz-Zadeh, j; Tajaddini-Sarmadi, J; Wolf, $\mathrm{S}$. 1994. Measurement of Plasma Hydro-peroxides Concentrations by Ferrous Oxidation-Xylenol Orange Assay in Conjuction with Triphenylphosphine. Analytical Biochem. 220, 403-409.

20. Araujo, A. L.; Radvanyi, F. 1987. Determination of phospholipase A2 activity by a colorimetric assay using a $\mathrm{pH}$ indicator. Toxicom, 25 (1) 181 -1188 .

21.Lowry, O. H.; Rosebrough, N. J.; Farr, A. L. y R. J. Randall. 1951. Protein measurement with the Folin-Phenol reagent. J. B. C. 193: 265-275.

22. Bragdon, J. 1951. Colorimetric determination of blood lipids. I. B. C. $190560-563$.
23.Agostoni, A.; Gerli, G.; Bertta, L.; Palazzini, G.; Buso, G. 1983. Antioxidants Enzymes and Selenium Serum Levels in an Andean Population. Clinical Chimica Acta 133: 153-157.

24.Gamblin, T.; King, M.; Kuret, J.; Berry, R.; Binder, L. 2000. Oxidative Regulation of Fatty AcidInduced Tau Polimerization. Biochem. 39(46), 14203-14210.

25.Mattson MP. 1998. Modification of ion homeostasis by lipid peroxidation: roles in neuronal degeneration and adaptative plasticity. Tends Neturose 21 (22), 53-57.

26. Braughler, J. M; Duncan, L; Chase, R. 1986 The Involvement fo Iron in Lipid Peroxidation. Importance of Ferric to Ferrous Ratios in Initiation. J. B. C. 261:10282-10289.

27.Rodríguez-Capote, K.; Céspedes, E.; Arencibia, R. 1998. Indicadores de Estrés Oxidativo en el Cerebro de Rata durante el Envejecimiento. Efecto del Factor de Crecimiento Nervioso. Rev. Neurol 27(157), 494-500.

28. Tibor Bakonyi and Zsolt Radak . 2004. High Altitude and Free Radicals. Review Article. Journal of Sports Science and Medicine 3, 64-69.

29. Vicente, M.; Miñano, M. 2002. Determinación de algunos antioxidantes en sujetos de altura. Trabajo de Aptitud Profesional para optar al título de Químico Farmacéutico. Facultad de Farmacia y Bioquímica, UNMSM. Lima. 